\title{
Approximate solutions of coupled Ramani equation by using RDTM with compared DTM and exact solutions
}

\author{
Murat Gubes ${ }^{1}$, Galip Oturanc ${ }^{2}$ \\ ${ }^{1}$ Department of Mathematics, Karamanoglu Mehmetbey University, Karaman, Turkey \\ ${ }^{2}$ Department of Mathematics, Selcuk University, Konya, Turkey
}

Received: 28 April 2016, Accepted: 21 July 2016

Published online: 12 November 2016.

\begin{abstract}
In this paper, we present a new approximate solutions of famous coupled Ramani Equation. In order to obtain the solution, we use the semi-analytical methods differential transform method (DTM) and reduced form of DTM called reduced differential transform method (RDTM). We compare the RDTM solutions with exact solution and DTM. Numerical results show clearly that DTM and RDTM are very effective and also provide very accurate solutions. Also, one can conclude that RDTM is used easier than DTM and converges faster than the DTM for these kind of problems.
\end{abstract}

Keywords: Reduced differential transform method (RDTM), differential transform method (DTM), coupled ramani equation, approximate solution.

\section{Introduction}

Partial differential equations are the fundamental of applied mathematics and they are frequently used in physic, engineering, chemistry and etc. In real life, many events can be modeled by a nonlinear partial differential equation such as evolution equations. Particularly in nonlinear sciences, one of the important and outstanding evolution equation is the famous coupled Ramani Equation that is presented as follow [1], [2], [3], [4].

$$
\begin{array}{r}
u(x, t)_{x x x x x x}+15 u(x, t)_{x x} u(x, t)_{x x x}+15 u(x, t)_{x} u(x, t)_{x x x x}+45\left(u(x, t)_{x}\right)^{2} u(x, t)_{x x} \\
-5 u(x, t)_{t t}+18 v(x, t)_{x}-5 u(x, t)_{x x x t}-15 u(x, t)_{x x} u(x, t)_{t}-15 u(x, t)_{x} u(x, t)_{x t}=0 \\
v(x, t)_{t}-v(x, t)_{x x x}-3 v(x, t)_{x} u(x, t)_{x}-3 v(x, t) u(x, t)_{x x}=0
\end{array}
$$

In literature, a great number of researchers have studied the system (1) to obtain exact and approximate solutions. Ablowitz and Clarkson [5], Ito [6], Zhang [7], Feng [8], Malfiet and Hereman [9] have investigated the solitons and inverse scattering, extensions, exact traveling wave solutions and traveling solitary wave solutions of nonlinear evolution equations respectively. Li has presented exact traveling wave solutions of six order Ramani and a coupled Ramani equation in [10]. In [11], Nadjafikhah and Shirvani-Sh have found Lie symmetries and conservation laws of Hirota-Ramani equation. Further, Yusufoglu and Bekir have obtained the two exact traveling wave solutions of coupled Ramani equation by applying tanh method as following [12]. 


$$
\begin{aligned}
& u(x, t)=a_{0}+2 \alpha \tanh (\alpha(x-\beta t)) \\
& v(x, t)=-\frac{4}{9} \beta \alpha^{4}-\frac{16}{27} \alpha^{6}+\frac{5}{9} \alpha^{2} \beta^{2}-\frac{5}{54} \beta^{3}+\left[\frac{20}{9} \beta \alpha^{4}+\frac{16}{9} \alpha^{6}-\frac{5}{9} \alpha^{2} \beta^{2}\right] \tanh ^{2}(\alpha(x-\beta t))
\end{aligned}
$$

and

$$
\begin{aligned}
& u(x, t)=a_{0}-2 \alpha \tan (\alpha(x-\beta t)) \quad\left(|\alpha(x-\beta t)|<\frac{\pi}{2}\right) \\
& v(x, t)=-\frac{4}{9} \beta \alpha^{4}+\frac{16}{27} \alpha^{6}-\frac{5}{9} \alpha^{2} \beta^{2}-\frac{5}{54} \beta^{3}+\left[-\frac{20}{9} \beta \alpha^{4}+\frac{16}{9} \alpha^{6}-\frac{5}{9} \alpha^{2} \beta^{2}\right] \tan ^{2}(\alpha(x-\beta t))
\end{aligned}
$$

where $a_{0}, \alpha$ and $\beta$ are arbitrary constants.

Recently, Wazwaz and Triki [13], Wazwaz [14], Jafarian et all [15] and Wazwaz [16] have presented the multiple soliton solutions and approximate solution of eq. (1) respectively.

The main goal of this study is to obtain accurate, convergent and efficient approximate solution of coupled Ramani equation (1) by using differential transform (DTM) and reduced differential transform (RDTM) methods. For the purpose of efficiency and accuracy, our results are compared with exact solutions (2) and (3). Numerical considerations are revealed that RDTM is very effective and more convergent than DTM. In addition, RDTM can be applied easier than DTM and ensures very accurate solutions as shown in Table (3)-(8) and Fig. (1)-(4). Moreover, RDTM is also faster than DTM in point of CPU times of computational process as seen in Table (9).

\section{Basic properties of two dimensional reduced differential transform method (RDTM) and differential transform method (DTM)}

\subsection{Two dimensional DTM}

Differential transform method (DTM) is a numerical method based on Taylor expansion. This method is related to find coefficients of series expansion of unknown function term by term. The concept of DTM was first proposed by Zhou [17]. By, considering the literature [17]-[26], we give the following definition of two dimensional DTM;

Definition 1. Let $u(x, t)$ be an analytic differentiable function, then two dimensional transform is follow

$$
U(k, h)=\frac{1}{k ! h !}\left[\frac{\partial^{k+h}}{\partial x^{k} \partial t^{h}} u(x, t)\right]_{x=x_{0}, t=t_{0}}
$$

where $U(k, h)$ is the transformed function of $u(x, t)$. The transformation is called T-function. Hence, the differential inverse transform of $U(k, h)$ is defined as

$$
u(x, t)=\sum_{k=0}^{\infty} \sum_{h=0}^{\infty} U(k, h)\left(x-x_{0}\right)^{k}\left(t-t_{0}\right)^{h}
$$

From the eqs. (4) and (5), it can be written

$$
u(x, t)=\sum_{k=0}^{\infty} \sum_{h=0}^{\infty} \frac{1}{k ! h !}\left[\frac{\partial^{k+h}}{\partial x^{k} \partial t^{h}} u(x, t)\right]_{x=x_{0}, t=t_{0}}\left(x-x_{0}\right)^{k}\left(t-t_{0}\right)^{h}
$$


In terms of applicability, we rearrange the eq. (6) as follow

$$
u(x, t)=\sum_{k=0}^{n} \sum_{h=0}^{m} \frac{1}{k ! h !}\left[\frac{\partial^{k+h}}{\partial x^{k} \partial t^{h}} u(x, t)\right]_{x=0, t=0} x^{k} t^{h}+R_{n m}(x, t)
$$

where $\left(x_{0}, t_{0}\right)$ are taken as $(0,0)$ and $R_{n m}(x, t)=\sum_{k=n+1}^{\infty} \sum_{h=m+1}^{\infty} U(k, h) x^{k} t^{h}$. Here, $R_{n m}(x, t)$ is negligibly small terms. Some of the transform form of functions are given as Table 1 and their proofs can be found in [17]-[20].

Table 1: Some two dimensional DTM operations with transformed forms.

\begin{tabular}{|c|c|}
\hline $\begin{array}{c}\text { Original functions } \\
u(x, t)=v(x, t) \pm w(x, t)\end{array}$ & $\begin{array}{c}\text { Transformed forms } \\
U(k, h)=V(k, h) \pm W(k, h)\end{array}$ \\
\hline$u(x, t)=\lambda v(x, t)$ & $U(k, h)=\lambda V(k, h)$ \\
\hline$u(x, t)=\frac{\partial}{\partial x} v(x, t)$ & $U(k, h)=(k+1) V(k+1, h)$ \\
\hline$u(x, t)=\frac{\partial}{\partial t} v(x, t)$ & $U(k, h)=(h+1) V(k, h+1)$ \\
\hline$u(x, t)=\frac{\partial^{m+n}}{\partial x^{m} \partial t^{n}} v(x, t)$ & $U(k, h)=\frac{(k+m) !}{k !} \frac{(h+n) !}{h !} V(k+m, h+n)$ \\
\hline$u(x, t)=v(x, t) w(x, t)$ & $U(k, h)=\sum_{r=0}^{k} \sum_{s=0}^{h} V(r, h-s) W(k-r, s)$ \\
\hline$u(x, t)=v(x, t) w(x, t) q(x, t)$ & $U(k, h)=\sum_{r=0}^{k} \sum_{p=0}^{k-r} \sum_{s=0}^{h} \sum_{z=0}^{h-s} V(r, h-s-z) W(p, s) Q(k-r-p, z)$ \\
\hline$u(x, t)=x^{m} t^{n}$ & $U(k, h)=\delta(k-m, h-n)=\delta(k-m) \delta(h-n), \quad \delta(k-m)= \begin{cases}1 & k=m \\
0 & \text { otherwise }\end{cases}$ \\
\hline
\end{tabular}

\subsection{Two dimensional RDTM}

Reduced differential transform method (RDTM) which has an alternative approach of problems is presented to overcome the demerit complex calculation, discretization, linearization or perturbations of well-known numerical and analytical methods such as Adomian decomposition, Differential transform, Homotopy perturbation and Variational iteration. RDTM was first introduced by Keskin and Oturanc [28]-[31]. The main advantage of RDTM is providing an analytic approximation, in many cases exact solutions, in rapidly convergent sequence with elegantly computed terms [26]-[39]. And also, unlike the DTM, RDTM is based on the Poisson series coefficients expansion. By using the literature [26]-[39], we present the RDTM as follow.

Definition 2. Let $u(x, t)$ be an analytic differentiable function and assumed that it can be demonstrated as a product of two functions which are single variable $u(x, t)=h(x) g(t)$. By making use of differential transform properties, $u(x, t)$ can be written as

$$
u(x, t)=\sum_{i=0}^{\infty} H(i) x^{i} \sum_{j=0}^{\infty} G(j) t^{j}=\sum_{k=0}^{\infty} U_{k}(x) t^{k}
$$

Here $U_{k}(x)$ is called dimensional spectrum function of $u(x, t)$. If function $u(x, t)$ is analytic and differentiated continuously with respect to time $t$ and space $x$ in the domain of interest, than

$$
U_{k}(x)=\frac{1}{k !}\left[\frac{\partial^{k}}{\partial t^{k}} u(x, t)\right]_{t=t_{0}}
$$


where $U_{k}(x)$ is transformed function of $u(x, t)$. The differential inverse transform of $U_{k}(x)$ is defined as

$$
u(x, t)=\sum_{k=0}^{\infty} U_{k}(x)\left(t-t_{0}\right)^{k} .
$$

Combining (8)-(10), we can write

$$
u(x, t)=\sum_{k=0}^{\infty} \frac{1}{k !}\left[\frac{\partial^{k}}{\partial t^{k}} u(x, t)\right]_{t=t_{0}}\left(t-t_{0}\right)^{k} .
$$

In real applications, we use the finite series form of (11), therefore we rewrite the solution as

$$
\tilde{u}_{n}(x, t)=\sum_{k=0}^{n} U_{k}(x) t^{k}
$$

where $n$ is order of approximation. Hence, the RDTM solution is given by

$$
u(x, t)=\lim _{n \rightarrow \infty} \tilde{u}_{n}(x, t)
$$

here $n$ is taken as sufficiently big to get convergent solution. In Table 2, transformed form of mathematical operation of some functions are given and their proofs are shown in ref. [28], [29].

Table 2: Some two dimensional RDTM operations with transformed forms.

\begin{tabular}{|c|c|}
\hline Original functions & Transformed forms \\
\hline$u(x, t)=v(x, t) \pm w(x, t)$ & $U_{k}(x)=V_{k}(x) \pm W_{k}(x)$ \\
\hline$u(x, t)=\lambda v(x, t)$ & $U_{k}(x)=\lambda V_{k}(x)$ \\
\hline$u(x, t)=\frac{\partial}{\partial x} v(x, t)$ & $U_{k}(x)=\frac{\partial}{\partial x} V_{k}(x)$ \\
\hline$u(x, t)=\frac{\partial^{r}}{\partial t^{r}} v(x, t)$ & $U_{k}(x)=\frac{(k+r) !}{k !} V_{k+r}(x)$ \\
\hline$u(x, t)=v(x, t) w(x, t)$ & $U_{k}(x)=\sum_{r=0}^{k} V_{r}(x) W_{k-r}(x)=\sum_{r=0}^{k} W_{r}(x) V_{k-r}(x)$ \\
\hline$u(x, t)=v(x, t) w(x, t) q(x, t)$ & $U_{k}(x)=\sum_{r=0}^{k} \sum_{p=0}^{k-r} V_{r}(x) W_{p}(x) Q_{k-r-p}(x)$ \\
\hline$u(x, t)=x^{m} t^{n}$ & $U_{k}(x)=x^{m} \delta(k-n), \quad \delta(k-n)= \begin{cases}x^{m} & k=n \\
0 & \text { otherwise }\end{cases}$ \\
\hline
\end{tabular}

\section{Solution procedures of Ramani equation by DTM and RDTM}

\subsection{DTM methodology}

Let's consider the coupled Ramani equation (1) with two different initial conditions as [4], [10],[12]-[16],

$$
\begin{aligned}
& u(x, 0)=a_{0}+2 \alpha \tanh (\alpha x) \\
& v(x, 0)=-\frac{4}{9} \beta \alpha^{4}-\frac{16}{27} \alpha^{6}+\frac{5}{9} \alpha^{2} \beta^{2}-\frac{5}{54} \beta^{3}+\left[\frac{20}{9} \beta \alpha^{4}+\frac{16}{9} \alpha^{6}-\frac{5}{9} \alpha^{2} \beta^{2}\right] \tanh ^{2}(\alpha x)
\end{aligned}
$$


and

$$
\begin{aligned}
& u(x, 0)=a_{0}-2 \alpha \tan (\alpha x) \\
& v(x, 0)=-\frac{4}{9} \beta \alpha^{4}+\frac{16}{27} \alpha^{6}-\frac{5}{9} \alpha^{2} \beta^{2}-\frac{5}{54} \beta^{3}+\left[-\frac{20}{9} \beta \alpha^{4}+\frac{16}{9} \alpha^{6}-\frac{5}{9} \alpha^{2} \beta^{2}\right] \tan ^{2}(\alpha x) .
\end{aligned}
$$

$U(k, h), V(k, h)$, which are called T-function, denote the transformation of the functions $u(x, t), v(x, t)$ in eq. (1) respectively. Then from Table (1) and eqs. (4) to (7), we obtain the transformed form of eq. (1) as below

$$
\begin{aligned}
& 5(h+1)(h+2) U(k, h+2)=\frac{(k+6) !}{k !} U(k+6, h)+18(k+1) V(k+1, h) \\
& +15 \sum_{r=0}^{k} \sum_{s=0}^{h}(r+1)(k-r+1)(k-r+2)(k-r+3)(k-r+4) U(r+1, h-s) U(k-r+4, s) \\
& +15 \sum_{r=0}^{k} \sum_{s=0}^{h}(r+1)(r+2)(k-r+1)(k-r+2)(k-r+3) U(r+2, h-s) U(k-r+3, s) \\
& -5 \frac{(k+3) !}{k !}(h+1) U(k+3, h+1)-15 \sum_{r=0}^{k} \sum_{=0}^{h}(h-s+1)(k-r+1)(k-r+2) U(k-r+2, s) U(r, h-s+1) \\
& -15 \sum_{r=0}^{k} \sum_{s=0}^{h}(r+1)(k-r+1)(h-s+1) U(r+1, h-s) U(k-r+1, h-s+1) \\
& +45 \sum_{r=0}^{k} \sum_{l=0}^{k-r} \sum_{s=0}^{h} \sum_{p=0}^{h-s}(r+1)(l+1)(k-r-l+1)(k-r-l+2) U(r+1, h-s-p) U(l+1, s) U(k-r-l+2, p) \\
& (h+1) V(k, h+1)=\frac{(k+3) !}{k !} V(k+3, h)+3 \sum_{r=0}^{k} \sum_{s=0}^{h}(r+1)(k-r+1) V(r+1, h-s) U(k-r+1, s) \\
& +3 \sum_{r=0}^{k} \sum_{s=0}^{h}(k-r+1)(k-r+2) V(r, h-s) U(k-r+2, s)
\end{aligned}
$$

and for initial conditions (14), (15), we obtain as

$$
\begin{aligned}
& U(k, 0)=a_{0} \delta(k, 0)+2 \alpha\left[\frac{(2 \alpha)^{k}-k ! \delta(k, 0)}{(2 \alpha)^{k}+k ! \delta(k, 0)}\right] \\
& V(k, 0)=\left(-\frac{4}{9} \beta \alpha^{4}-\frac{16}{27} \alpha^{6}+\frac{5}{9} \alpha^{2} \beta^{2}-\frac{5}{54} \beta^{3}\right) \delta(k, 0)+\left(\frac{20}{9} \beta \alpha^{4}+\frac{16}{9} \alpha^{6}-\frac{5}{9} \alpha^{2} \beta^{2}\right)\left(\frac{(2 \alpha)^{k}-k ! \delta(k, 0)}{(2 \alpha)^{k}+k ! \delta(k, 0)}\right)^{2}
\end{aligned}
$$

and

$$
\begin{aligned}
& U(k, 0)=a_{0} \delta(k, 0)-2 \alpha \tan \left(\frac{k \pi}{2}\right) \\
& V(k, 0)=\left(-\frac{4}{9} \beta \alpha^{4}+\frac{16}{27} \alpha^{6}-\frac{5}{9} \alpha^{2} \beta^{2}-\frac{5}{54} \beta^{3}\right) \delta(k, 0)+\left(-\frac{20}{9} \beta \alpha^{4}+\frac{16}{9} \alpha^{6}-\frac{5}{9} \alpha^{2} \beta^{2}\right)\left(\tan \left(\frac{k \pi}{2}\right)\right)^{2}
\end{aligned}
$$

We put firstly (18) into (16)-(17) and using the DTM, we get the three terms approximate traveling DTM solution of coupled Ramani equation as 


$$
\begin{aligned}
U_{3,3}(x, t) & =a_{0}+2 \alpha^{2} x-2 / 3 \alpha^{4} x^{3}-2 \beta \alpha^{2} t+2 \beta \alpha^{4} x^{2} t-2 \alpha^{4} \beta^{2} x t^{2}+8 / 3 \alpha^{6} \beta^{2} x^{3} t^{2}+\left(-4 \alpha^{6} \beta^{2}+2 / 3 \alpha^{4} \beta^{3}\right) t^{3} \\
& +\left(-4 \beta \alpha^{6}+8 \alpha^{8} \beta^{3}\right) x t^{3}+\left(28 \alpha^{8} \beta^{2}-8 / 3 \alpha^{6} \beta^{3}\right) x^{2} t^{3}+\left(\frac{28}{3} \beta \alpha^{8}-\frac{128}{3} \alpha^{10} \beta^{3}\right) x^{3} t^{3} \\
V_{3,3}(x, t) & =-4 / 9 \beta \alpha^{4}-\frac{16}{27} \alpha^{6}+5 / 9 \beta^{2} \alpha^{2}-\frac{5}{54} \beta^{3}+\left(\frac{20}{9} \beta \alpha^{4}+\frac{16}{9} \alpha^{6}-5 / 9 \beta^{2} \alpha^{2}\right) \alpha^{2} x^{2} \\
& +\left(-\frac{32}{9} \beta \alpha^{8}-\frac{40}{9} \alpha^{6} \beta^{2}+\frac{10}{9} \alpha^{4} \beta^{3}\right) x t+\left(\frac{128}{27} \beta \alpha^{10}+\frac{160}{27} \alpha^{8} \beta^{2}-\frac{40}{27} \alpha^{6} \beta^{3}\right) x^{3} t \\
& +\left(\frac{16}{9} \alpha^{8} \beta^{2}+\frac{20}{9} \alpha^{6} \beta^{3}-5 / 9 \beta^{4} \alpha^{4}\right) t^{2}+\left(-\frac{64}{9} \alpha^{10} \beta^{2}-\frac{80}{9} \alpha^{8} \beta^{3}+\frac{20}{9} \beta^{4} \alpha^{6}\right) x^{2} t^{2} \\
& +\left(\frac{128}{27} \alpha^{10} \beta^{3}+\frac{160}{27} \beta^{4} \alpha^{8}-\frac{40}{27} \alpha^{6} \beta^{5}\right) x t^{3}+\left(-\frac{1360}{81} \beta^{4} \alpha^{10}+\frac{340}{81} \alpha^{8} \beta^{5}-\frac{1088}{81} \alpha^{12} \beta^{3}\right) x^{3} t^{3}
\end{aligned}
$$

and secondly put (19) into (16)-(17), we obtain the other traveling DTM solution of eq. (1) as following

$$
\begin{aligned}
U_{3,3}(x, t) & =a_{0}-2 \alpha^{2} x-2 / 3 \alpha^{4} x^{3}+2 \alpha^{2} \beta t+2 \beta \alpha^{4} x^{2} t-2 \alpha^{4} \beta^{2} x t^{2}-8 / 3 \alpha^{6} \beta^{2} x^{3} t^{2}+\left(4 \alpha^{6} \beta^{2}+2 / 3 \alpha^{4} \beta^{3}\right) t^{3} \\
& +\left(4 \alpha^{6} \beta+8 \alpha^{8} \beta^{3}\right) x t^{3}+\left(28 \alpha^{8} \beta^{2}+8 / 3 \alpha^{6} \beta^{3}\right) x^{2} t^{3}+\left(\frac{28}{3} \alpha^{8} \beta+\frac{128}{3} \alpha^{10} \beta^{3}\right) x^{3} t^{3} \\
V_{3,3}(x, t) & =-4 / 9 \beta \alpha^{4}+\frac{16}{27} \alpha^{6}-5 / 9 \beta^{2} \alpha^{2}-\frac{5}{54} \beta^{3}+\left(-\frac{20}{9} \beta \alpha^{4}+\frac{16}{9} \alpha^{6}-5 / 9 \beta^{2} \alpha^{2}\right) \alpha^{2} x^{2} \\
& +\left(-\frac{32}{9} \alpha^{8} \beta+\frac{40}{9} \alpha^{6} \beta^{2}+\frac{10}{9} \alpha^{4} \beta^{3}\right) x t+\left(-\frac{128}{27} \alpha^{10} \beta+\frac{160}{27} \alpha^{8} \beta^{2}+\frac{40}{27} \alpha^{6} \beta^{3}\right) x^{3} t \\
& +\left(\frac{16}{9} \alpha^{8} \beta^{2}-\frac{20}{9} \alpha^{6} \beta^{3}-5 / 9 \beta^{4} \alpha^{4}\right) t^{2}+\left(\frac{64}{9} \alpha^{10} \beta^{2}-\frac{80}{9} \alpha^{8} \beta^{3}-\frac{20}{9} \alpha^{6} \beta^{4}\right) x^{2} t^{2} \\
& +\left(-\frac{128}{27} \alpha^{10} \beta^{3}+\frac{160}{27} \alpha^{8} \beta^{4}+\frac{40}{27} \alpha^{6} \beta^{5}\right) x t^{3}+\left(\frac{1360}{81} \alpha^{10} \beta^{4}+\frac{340}{81} \alpha^{8} \beta^{5}-\frac{1088}{81} \alpha^{12} \beta^{3}\right) x^{3} t^{3}
\end{aligned}
$$

Hence, it is clearly seen in Table (3) to (8) that solutions (20) and (21) provide the good accuracy with compared exact solutions [12].

Table 3: For $u(x, t)$, numerical results of seven steps DTM and RDTM solutions of eq. (1) with compared exact solution (2) at $t=20$ and $a_{0}=1, \alpha=\beta=0.01$.

\begin{tabular}{|c|c|c|c|c|c|}
\hline$x$ & $\begin{array}{c}\text { Exact[12] } \\
u(x, t)\end{array}$ & $\begin{array}{c}\text { RDTM } \\
U_{7}(x)\end{array}$ & $\begin{array}{c}\text { DTM } \\
U_{7,7}(x, t)\end{array}$ & $\begin{array}{c}\text { Errors of RDTM } \\
\left|u(x, t)-U_{7}(x)\right|\end{array}$ & $\begin{array}{c}\text { Errors of DTM } \\
\left|u(x, t)-U_{7,7}(x, t)\right|\end{array}$ \\
\hline-50 & 0.990726228 & 0.9907262252 & 0.9909484041 & $2.8 \times 10^{-9}$ & $2.221761 \times 10^{-4}$ \\
\hline-40 & 0.9923668212 & 0.9923668186 & 0.9925853005 & $2.6 \times 10^{-9}$ & $2.184793 \times 10^{-4}$ \\
\hline-30 & 0.9941371636 & 0.9941371613 & 0.994329091 & $2.3 \times 10^{-9}$ & $1.919273 \times 10^{-4}$ \\
\hline-20 & 0.9960140671 & 0.9960140653 & 0.9961574997 & $1.8 \times 10^{-9}$ & $1.434326 \times 10^{-4}$ \\
\hline-10 & 0.9979670454 & 0.9979670444 & 0.9980439141 & $1.0 \times 10^{-9}$ & $7.68687 \times 10^{-5}$ \\
\hline 10 & 1.001953749 & 1.00195375 & 1.001876877 & $1.0 \times 10^{-9}$ & $7.6872 \times 10^{-5}$ \\
\hline 20 & 1.003909050 & 1.003909051 & 1.003765614 & $1.0 \times 10^{-9}$ & $1.43436 \times 10^{-4}$ \\
\hline 30 & 1.005789625 & 1.005789628 & 1.005597698 & $3.0 \times 10^{-9}$ & $1.91927 \times 10^{-4}$ \\
\hline 40 & 1.007564728 & 1.00756473 & 1.007346259 & $2.0 \times 10^{-9}$ & $2.18469 \times 10^{-4}$ \\
\hline 50 & 1.009210856 & 1.009210859 & 1.008988739 & $3.0 \times 10^{-9}$ & $2.22117 \times 10^{-4}$ \\
\hline
\end{tabular}



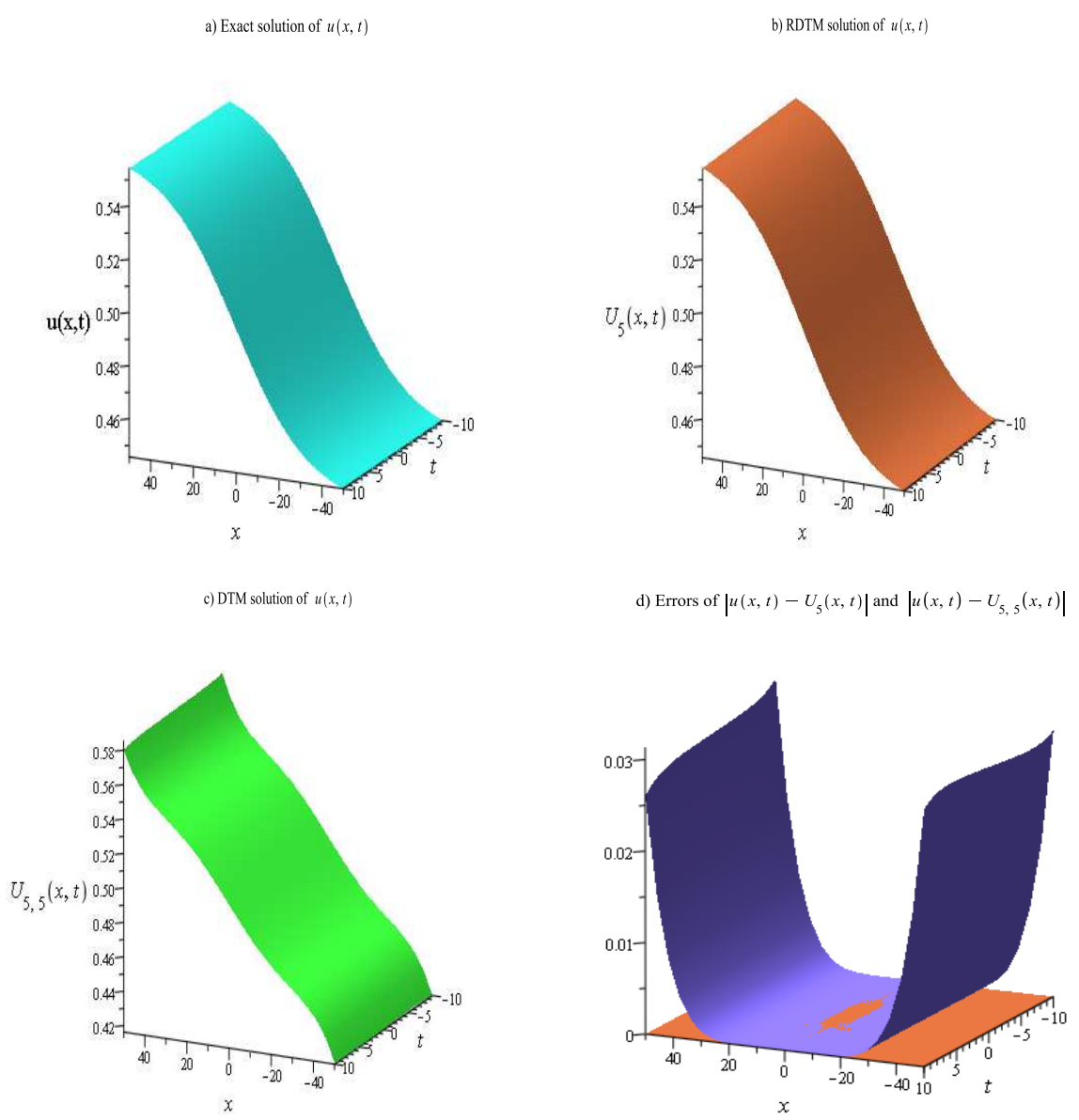

Fig. 1: Comparison between exact solution (2) and five steps RDTM, DTM solutions of $u(x, t)$ at $a_{0}=\frac{1}{2}, \alpha=\beta=0.03$. And comparison of errors d) Sienna-RDTM, SlateBlue-DTM.

Table 4: For $v(x, t)$, numerical results of seven steps DTM and RDTM solutions of eq. (1) with compared exact solution (2) at $t=20$ and $a_{0}=1, \alpha=\beta=0.01$.

\begin{tabular}{|c|c|c|c|c|c|}
\hline$x$ & $\begin{array}{c}\text { Exact[12] } \\
v(x, t)\end{array}$ & $\begin{array}{c}\text { RDTM } \\
V_{7}(x)\end{array}$ & $\begin{array}{c}\text { DTM } \\
V_{7,7}(x, t)\end{array}$ & $\begin{array}{c}\text { Errors of RDTM } \\
\left|v(x, t)-V_{7}(x)\right|\end{array}$ & $\begin{array}{c}\text { Errors of DTM } \\
\left|v(x, t)-V_{7,7}(x, t)\right|\end{array}$ \\
\hline-50 & $-8.822839648 \times 10^{-8}$ & $-8.822106112 \times 10^{-8}$ & $-8.823201645 \times 10^{-8}$ & $7.33534 \times 10^{-12}$ & $3.61999 \times 10^{-12}$ \\
\hline-40 & $-8.785868735 \times 10^{-8}$ & $-8.785217836 \times 10^{-8}$ & $-8.785931076 \times 10^{-8}$ & $6.50899 \times 10^{-12}$ & $6.2341 \times 10^{-13}$ \\
\hline-30 & $-8.754022597 \times 10^{-8}$ & $-8.753492301 \times 10^{-8}$ & $-8.754027998 \times 10^{-8}$ & $5.30295 \times 10^{-12}$ & $5.401 \times 10^{-14}$ \\
\hline-20 & $-8.729383894 \times 10^{-8}$ & $-8.729008043 \times 10^{-8}$ & $-8.729383182 \times 10^{-8}$ & $3.75851 \times 10^{-12}$ & $7.12 \times 10^{-15}$ \\
\hline-10 & $-8.713716109 \times 10^{-8}$ & $-8.713520229 \times 10^{-8}$ & $-8.713715553 \times 10^{-8}$ & $1.9588 \times 10^{-12}$ & $5.561 \times 10^{-15}$ \\
\hline 10 & $-8.713295226 \times 10^{-8}$ & $-8.71348706 \times 10^{-8}$ & $-8.713295226 \times 10^{-8}$ & $1.91834 \times 10^{-12}$ & $5.59 \times 10^{-15}$ \\
\hline 20 & $-8.72857484 \times 10^{-8}$ & $-8.728947099 \times 10^{-8}$ & $-8.72857608 \times 10^{-8}$ & $3.72259 \times 10^{-12}$ & $1.24 \times 10^{-14}$ \\
\hline 30 & $-8.752885527 \times 10^{-8}$ & $-8.753412915 \times 10^{-8}$ & $-8.752893306 \times 10^{-8}$ & $5.27388 \times 10^{-12}$ & $7.779 \times 10^{-14}$ \\
\hline 40 & $-8.784482114 \times 10^{-8}$ & $-8.785130927 \times 10^{-8}$ & $-8.784547184 \times 10^{-8}$ & $6.48813 \times 10^{-12}$ & $6.507 \times 10^{-13}$ \\
\hline 50 & $-8.821289529 \times 10^{-8}$ & $-8.822021826 \times 10^{-8}$ & $-8.821656659 \times 10^{-8}$ & $7.32297 \times 10^{-12}$ & $3.6713 \times 10^{-12}$ \\
\hline
\end{tabular}



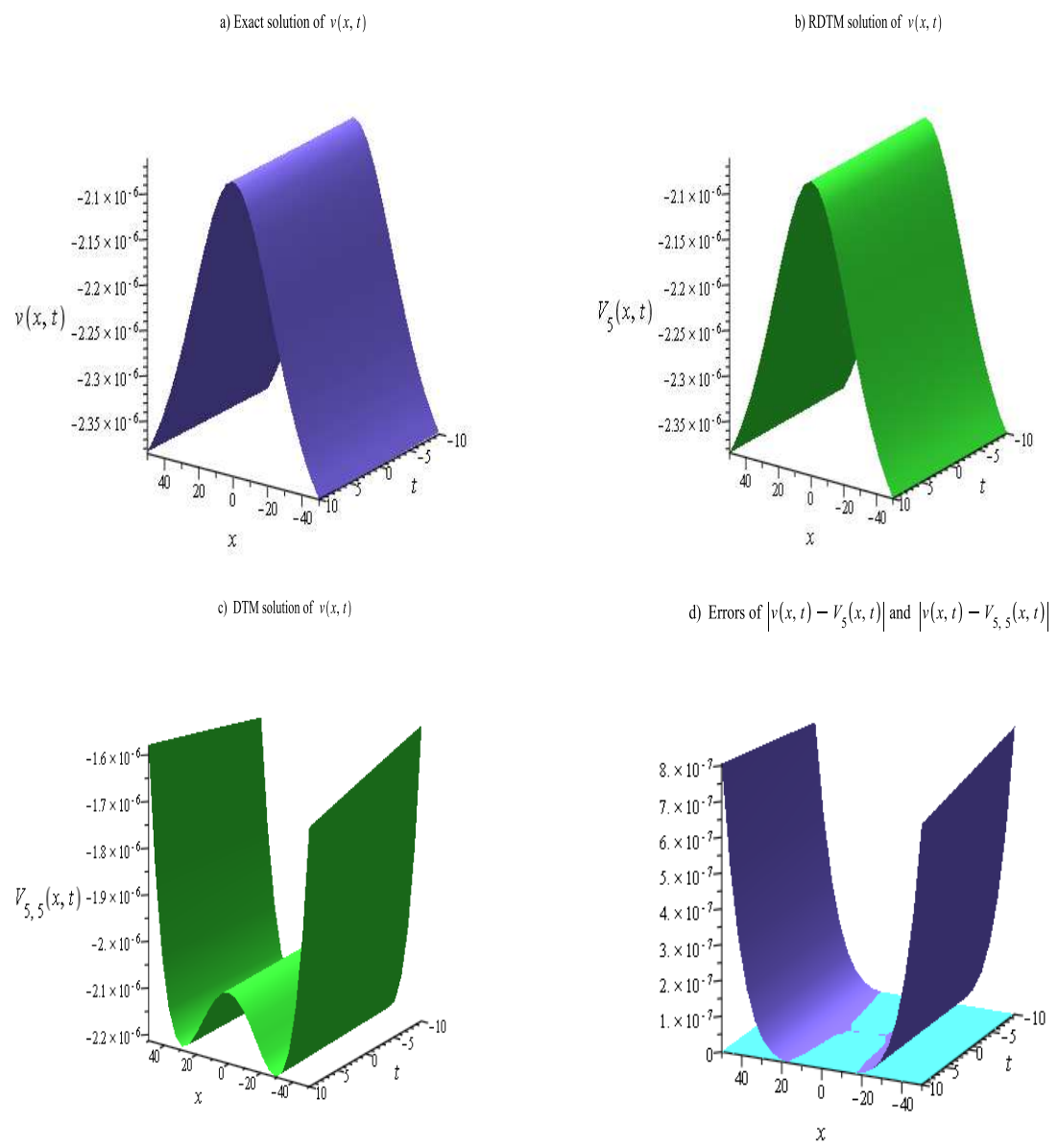

Fig. 2: Comparison between exact solution (2) and five steps RDTM, DTM solutions of $v(x, t)$ at $a_{0}=\frac{1}{2}, \alpha=\beta=0.03$. And comparison of errors d) Medium Turquoise-RDTM, SlateBlue-DTM.

\subsection{RDTM methodology}

As the same manner, again we consider the eq. (1) with initial conditions (14)-(15) to obtain the RDTM solutions. $U_{k}(x)$, $V_{k}(x)$, which are called $t$ dimensional spectrum functions, denote the transformation of the functions $u(x, t), v(x, t$ in eq. (1) respectively. Then from Table 2 and eqs. (8) to (13), we obtain the transformed form of eq. (1) as below

$$
\begin{aligned}
& 5(k+1)(k+2) U_{k+2}(x)=\frac{d^{6}}{d x^{6}} U_{k}(x)+18 \frac{d}{d x} V_{k}(x)+15 \sum_{r=0}^{k} \frac{d^{2}}{d x^{2}} U_{k-r}(x) \frac{d^{3}}{d x^{3}} U_{r}(x)+15 \sum_{r=0}^{k} \frac{d}{d x} U_{k-r}(x) \frac{d^{4}}{d x^{4}} U_{r}(x) \\
&-15 \sum_{r=0}^{k} \frac{d^{2}}{d x^{2}} U_{r}(x)(k-r+1) U_{k-r+1}(x)-15 \sum_{r=0}^{k} \frac{d}{d x} U_{r}(x)(k-r+1) \frac{d}{d x} U_{k-r+1}(x) \\
&+45 \sum_{r=0}^{k} \sum_{s=0}^{k-r} \frac{d}{d x} U_{r}(x) \frac{d}{d x} U_{s}(x) \frac{d^{2}}{d x^{2}} U_{k-r-s}(x)-5(k+1) \frac{d^{3}}{d x^{3}} U_{k+1}(x) \\
&(k+1) V_{k+1}(x)=\frac{d^{3}}{d x^{3}} V_{k}(x)+3 \sum_{r=0}^{k} \frac{d}{d x} V_{r}(x) \frac{d}{d x} U_{k-r}(x)+3 \sum_{r=0}^{k} V_{r}(x) \frac{d^{2}}{d x^{2}} U_{k-r}(x)
\end{aligned}
$$


and for initial conditions (14)-(15), we obtain reduced transform form as respectively

$$
\begin{aligned}
& U_{0}(x)=a_{0}+2 \alpha \tanh (\alpha x) \\
& V_{0}(x)=\left(-\frac{4}{9} \beta \alpha^{4}-\frac{16}{27} \alpha^{6}+\frac{5}{9} \alpha^{2} \beta^{2}-\frac{5}{54} \beta^{3}\right)+\left(\frac{20}{9} \beta \alpha^{4}+\frac{16}{9} \alpha^{6}-\frac{5}{9} \alpha^{2} \beta^{2}\right) \tanh ^{2}(\alpha x)
\end{aligned}
$$

and

$$
\begin{aligned}
& U_{0}(x)=a_{0}-2 \alpha \tan (\alpha x) \\
& V_{0}(x)=\left(-\frac{4}{9} \beta \alpha^{4}+\frac{16}{27} \alpha^{6}-\frac{5}{9} \alpha^{2} \beta^{2}-\frac{5}{54} \beta^{3}\right)+\left(-\frac{20}{9} \beta \alpha^{4}+\frac{16}{9} \alpha^{6}-\frac{5}{9} \alpha^{2} \beta^{2}\right) \tan ^{2}(\alpha x)
\end{aligned}
$$

Table 5: For $u(x, t)$, numerical results of seven steps DTM and RDTM solutions of eq. (1) with compared exact solution (3) at $t=20$ and $a_{0}=1, \alpha=\beta=0.01$.

\begin{tabular}{|c|c|c|c|c|c|}
\hline$x$ & $\begin{array}{c}\text { Exact[12] } \\
u(x, t)\end{array}$ & $\begin{array}{c}\text { RDTM } \\
U_{7}(x)\end{array}$ & $\begin{array}{c}\text { DTM } \\
U_{7,7}(x, t)\end{array}$ & $\begin{array}{c}\text { Errors of RDTM } \\
\left|u(x, t)-U_{7}(x)\right|\end{array}$ & $\begin{array}{c}\text { Errors of DTM } \\
\left|u(x, t)-U_{7,7}(x, t)\right|\end{array}$ \\
\hline-50 & 1.010978045 & 1.010978054 & 1.010257505 & $9.0 \times 10^{-9}$ & $7.2054 \times 10^{-4}$ \\
\hline-40 & 1.008503055 & 1.00850306 & 1.008041336 & $5.0 \times 10^{-9}$ & $4.61719 \times 10^{-4}$ \\
\hline-30 & 1.006230580 & 1.006230583 & 1.005938409 & $3.0 \times 10^{-9}$ & $2.92171 \times 10^{-4}$ \\
\hline-20 & 1.004095861 & 1.004095864 & 1.003922985 & $3.0 \times 10^{-9}$ & $1.72876 \times 10^{-4}$ \\
\hline-10 & 1.002047104 & 1.002047105 & 1.001966559 & $1.0 \times 10^{-9}$ & $8.0545 \times 10^{-5}$ \\
\hline 10 & 0.9980337012 & 0.9980337003 & 0.9981142416 & $9.0 \times 10^{-10}$ & $8.05404 \times 10^{-5}$ \\
\hline 20 & 0.9959874261 & 0.995987424 & 0.9961602952 & $2.1 \times 10^{-9}$ & $1.728691 \times 10^{-4}$ \\
\hline 30 & 0.9938570755 & 0.9938570716 & 0.9941492333 & $3.9 \times 10^{-9}$ & $2.921578 \times 10^{-4}$ \\
\hline 40 & 0.9915912460 & 0.9915912401 & 0.9920529338 & $5.9 \times 10^{-9}$ & $4.616878 \times 10^{-4}$ \\
\hline 50 & 0.9891258314 & 0.9891258222 & 0.9898462633 & $9.2 \times 10^{-9}$ & $7.204319 \times 10^{-4}$ \\
\hline
\end{tabular}

Table 6: For $v(x, t)$, numerical results of seven steps DTM and RDTM solutions of eq. (1) with compared exact solution (3) at $t=20$ and $a_{0}=1, \alpha=\beta=0.01$.

\begin{tabular}{|c|c|c|c|c|c|}
\hline$x$ & $\begin{array}{c}\text { Exact[12] } \\
v(x, t)\end{array}$ & $\begin{array}{c}\text { RDTM } \\
V_{7}(x)\end{array}$ & $\begin{array}{c}\text { DTM } \\
V_{7,7}(x, t)\end{array}$ & $\begin{array}{c}\text { Errors of RDTM } \\
\left|v(x, t)-V_{7}(x)\right|\end{array}$ & $\begin{array}{c}\text { Errors of DTM } \\
\left|v(x, t)-V_{7,7}(x, t)\right|\end{array}$ \\
\hline-50 & $-9.993227215 \times 10^{-8}$ & $-9.991393181 \times 10^{-8}$ & $-9.992708042 \times 10^{-8}$ & $1.834034 \times 10^{-11}$ & $5.16173 \times 10^{-12}$ \\
\hline-40 & $-9.923603996 \times 10^{-8}$ & $-9.922331725 \times 10^{-8}$ & $-9.923517293 \times 10^{-8}$ & $1.272271 \times 10^{-11}$ & $8.6703 \times 10^{-13}$ \\
\hline-30 & $-9.875256259 \times 10^{-8}$ & $-9.874398369 \times 10^{-8}$ & $-9.875245418 \times 10^{-8}$ & $8.5789 \times 10^{-12}$ & $1.0841 \times 10^{-13}$ \\
\hline-20 & $-9.843424659 \times 10^{-8}$ & $-9.842893011 \times 10^{-8}$ & $-9.843422759 \times 10^{-8}$ & $5.31648 \times 10^{-12}$ & $1.9 \times 10^{-14}$ \\
\hline-10 & $-9.825251278 \times 10^{-8}$ & $-9.824995748 \times 10^{-8}$ & $-9.825250579 \times 10^{-8}$ & $2.5553 \times 10^{-12}$ & $6.99 \times 10^{-15}$ \\
\hline 10 & $-9.824782982 \times 10^{-8}$ & $-9.825033777 \times 10^{-8}$ & $-9.824783681 \times 10^{-8}$ & $2.50795 \times 10^{-12}$ & $6.99 \times 10^{-15}$ \\
\hline 20 & $-9.842449482 \times 10^{-8}$ & $-9.842975832 \times 10^{-8}$ & $-9.842450789 \times 10^{-8}$ & $5.2635 \times 10^{-12}$ & $1.307 \times 10^{-14}$ \\
\hline 30 & $-9.873690092 \times 10^{-8}$ & $-9.874541644 \times 10^{-8}$ & $-9.873685319 \times 10^{-8}$ & $8.51552 \times 10^{-12}$ & $4.773 \times 10^{-14}$ \\
\hline 40 & $-9.921301115 \times 10^{-8}$ & $-9.922565351 \times 10^{-8}$ & $-9.921226004 \times 10^{-8}$ & $1.264236 \times 10^{-11}$ & $7.5111 \times 10^{-13}$ \\
\hline 50 & $-9.98994945 \times 10^{-8}$ & $-9.99177279 \times 10^{-8}$ & $-9.989455455 \times 10^{-8}$ & $1.82334 \times 10^{-11}$ & $4.93995 \times 10^{-12}$ \\
\hline
\end{tabular}


As in the DTM solution process, by using RDTM algorithm we put firstly (24) into (22)-(23), we get the three terms RDTM solution of coupled Ramani equation as

$$
\begin{aligned}
U_{3}(x) & =a_{0}+2 \alpha \tanh (\alpha x)-2 \alpha^{2} \beta\left(1-\tanh ^{2}(\alpha x)\right) t-\frac{2 \sinh (\alpha x) \alpha^{3} t^{2}\left(-48 \alpha^{4} \cosh ^{2}(\alpha x)\right)}{\cosh ^{7}(\alpha x)} \\
& -\frac{2 \sinh (\alpha x) \alpha^{3} t^{2}\left(72 \alpha^{4}-12 \alpha^{2} \beta \cosh ^{2}(\alpha x)+\beta^{2} \cosh ^{4}(\alpha x)\right)}{\cosh ^{7}(\alpha x)} \\
& -\frac{32}{15 \cosh ^{10}(\alpha x)}\left[1260 \alpha^{8} \beta \cosh ^{4}(\alpha x)+8820 \alpha^{10} \cosh ^{4}(\alpha x)-945 \beta \alpha^{8} \cosh ^{2}(\alpha x)+11340 \alpha^{10}\right] t^{3} \\
& -\frac{32}{15 \cosh ^{10}(\alpha x)}\left[12 \alpha^{8} \beta \cosh ^{8}(\alpha x)-18900 \alpha^{10} \cosh ^{2}(\alpha x)\right] t^{3} \\
& -\frac{32}{15 \cosh ^{10}(\alpha x)}\left[-1020 \alpha^{10} \cosh ^{6}(\alpha x)+8 \alpha^{10} \cosh ^{8}(\alpha x)-378 \alpha^{8} \beta \cosh ^{6}(\alpha x)\right] t^{3}
\end{aligned}
$$

$$
\begin{aligned}
V_{3}(x) & =-\frac{4}{9} \beta \alpha^{4}-\frac{16}{27} \alpha^{6}+\frac{5}{9} \beta^{2} \alpha^{2}-\frac{5}{54} \beta^{3}+\left(\frac{20}{9} \beta \alpha^{4}+\frac{16}{9} \alpha^{6}-\frac{5}{9} \beta^{2} \alpha^{2}\right) \tanh ^{2}(\alpha x) \\
& +\frac{8}{9} \frac{\sinh (\alpha x)\left(\cosh ^{2}(\alpha x)-3\right)\left(20 \alpha^{2} \beta+16 \alpha^{4}-5 \beta^{2}\right) \alpha^{5} t}{\cosh ^{5}(\alpha x)} \\
& -\frac{9}{8} \frac{\alpha^{8}\left(20 \alpha^{2} \beta+16 \alpha^{4}-5 \beta^{2}\right)\left[4 \cosh ^{6}(\alpha x)-126 \cosh ^{4}(\alpha x)+420 \cosh ^{2}(\alpha x)-315\right] t^{2}}{\cosh ^{8}(\alpha x)} \\
& +\frac{128}{27} \frac{\left(6615 \cosh ^{4}(\alpha x)-18900 \cosh ^{2}(\alpha x)\right)\left[20 \alpha^{2} \beta+16 \alpha^{4}-5 \beta^{2}\right] \alpha^{11} \sinh (\alpha x) t^{3}}{\cosh ^{11}(\alpha x)} \\
& +\frac{128}{27} \frac{\left(2 \cosh ^{8}(\alpha x)-510 \cosh ^{6}(\alpha x)+14175\right)\left[20 \alpha^{2} \beta+16 \alpha^{4}-5 \beta^{2}\right] \alpha^{11} \sinh (\alpha x) t^{3}}{\cosh ^{11}(\alpha x)}
\end{aligned}
$$

and secondly put (25) into (22)-(23), we obtain the other RDTM solution of eq. (1) as following

$$
\begin{aligned}
U_{3}(x) & =a_{0}-2 \alpha \tan (\alpha x)+2 \alpha^{2} \beta\left(1+\tan ^{2}(\alpha x)\right) t-\frac{2 \sin (\alpha x) \alpha^{3} t^{2}\left(-48 \alpha^{4} \cos ^{2}(\alpha x)\right)}{\cos ^{7}(\alpha x)} \\
& -\frac{2 \sin (\alpha x) \alpha^{3} t^{2}\left(72 \alpha^{4}+12 \alpha^{2} \beta \cos ^{2}(\alpha x)+\beta^{2} \cos ^{4}(\alpha x)\right)}{\cos ^{7}(\alpha x)} \\
& +\frac{32}{15 \cos ^{10}(\alpha x)}\left[\left(8820 \alpha^{2} \cos ^{4}(\alpha x)+945 \beta \cos ^{2}(\alpha x)+378 \beta \cos ^{6}(\alpha x)+11340 \alpha^{2}\right) \alpha^{8}\right] t^{3} \\
& +\frac{32}{15 \cos ^{10}(\alpha x)}\left[\left(-1260 \beta \cos ^{4}(\alpha x)-1020 \alpha^{2} \cos ^{6}(\alpha x)-18900 \alpha^{2} \cos ^{2}(\alpha x)\right) \alpha^{8}\right] t^{3} \\
& +\frac{32}{15 \cos ^{10}(\alpha x)}\left[\left(-12 \beta \cos ^{8}(\alpha x)+8 \alpha^{2} \cos ^{8}(\alpha x)\right) \alpha^{8}\right] t^{3}
\end{aligned}
$$



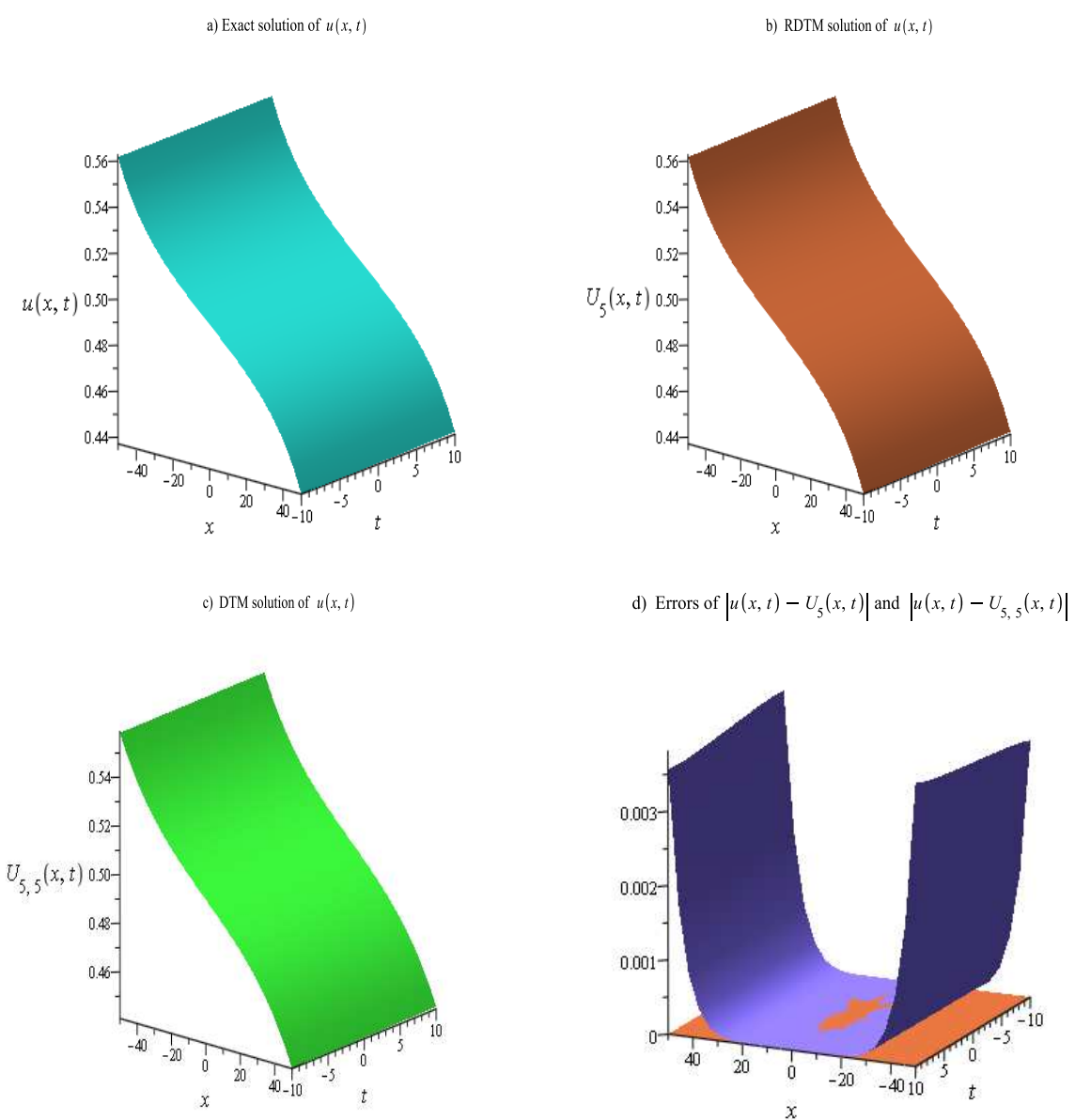

Fig. 3: Comparison between exact solution (3) and five steps RDTM, DTM solutions of $u(x, t)$ at $a_{0}=\frac{1}{2}, \alpha=\beta=0.02$. And comparison of errors d) Sienna-RDTM, SlateBlue-DTM.

$$
\begin{aligned}
V_{3}(x) & =-\frac{4}{9} \beta \alpha^{4}+\frac{16}{27} \alpha^{6}-\frac{5}{9} \beta^{2} \alpha^{2}-\frac{5}{54} \beta^{3}+\left(\frac{-20}{9} \beta \alpha^{4}+\frac{16}{9} \alpha^{6}-\frac{5}{9} \beta^{2} \alpha^{2}\right) \tan ^{2}(\alpha x) \\
& -\frac{8}{9} \frac{\sin (\alpha x) \alpha^{5} t\left(\cos ^{2}(\alpha x)-3\right)\left(-20 \alpha^{2} \beta+16 \alpha^{4}-5 \beta^{2}\right)}{\cos ^{5}(\alpha x)} \\
& -\frac{9}{8} \frac{\alpha^{8}\left(-20 \alpha^{2} \beta+16 \alpha^{4}-5 \beta^{2}\right)\left[-126 \cos ^{4}(\alpha x)+4 \cos ^{6}(\alpha x)+420 \cos ^{2}(\alpha x)-315\right] t^{2}}{\cos ^{8}(\alpha x)} \\
& +\frac{128}{27} \frac{\left(6615 \cos ^{4}(\alpha x)-18900 \cos ^{2}(\alpha x)\right)\left[-20 \alpha^{2} \beta+16 \alpha^{4}-5 \beta^{2}\right] \alpha^{11} \sin (\alpha x) t^{3}}{\cos ^{11}(\alpha x)} \\
& +\frac{128}{27} \frac{\left(2 \cos ^{8}(\alpha x)-510 \cos ^{6}(\alpha x)+14175\right)\left[-20 \alpha^{2} \beta+16 \alpha^{4}-5 \beta^{2}\right] \alpha^{11} \sin (\alpha x) t^{3}}{\cos ^{11}(\alpha x)}
\end{aligned}
$$

Thus, it is obviously noted on the Table 3 to 8 and Fig. (1)-(4) that solutions (26)-(29) provide the good accuracy with compared exact [12] and DTM solutions. Also from Table 9, RDTM is more faster than DTM. 

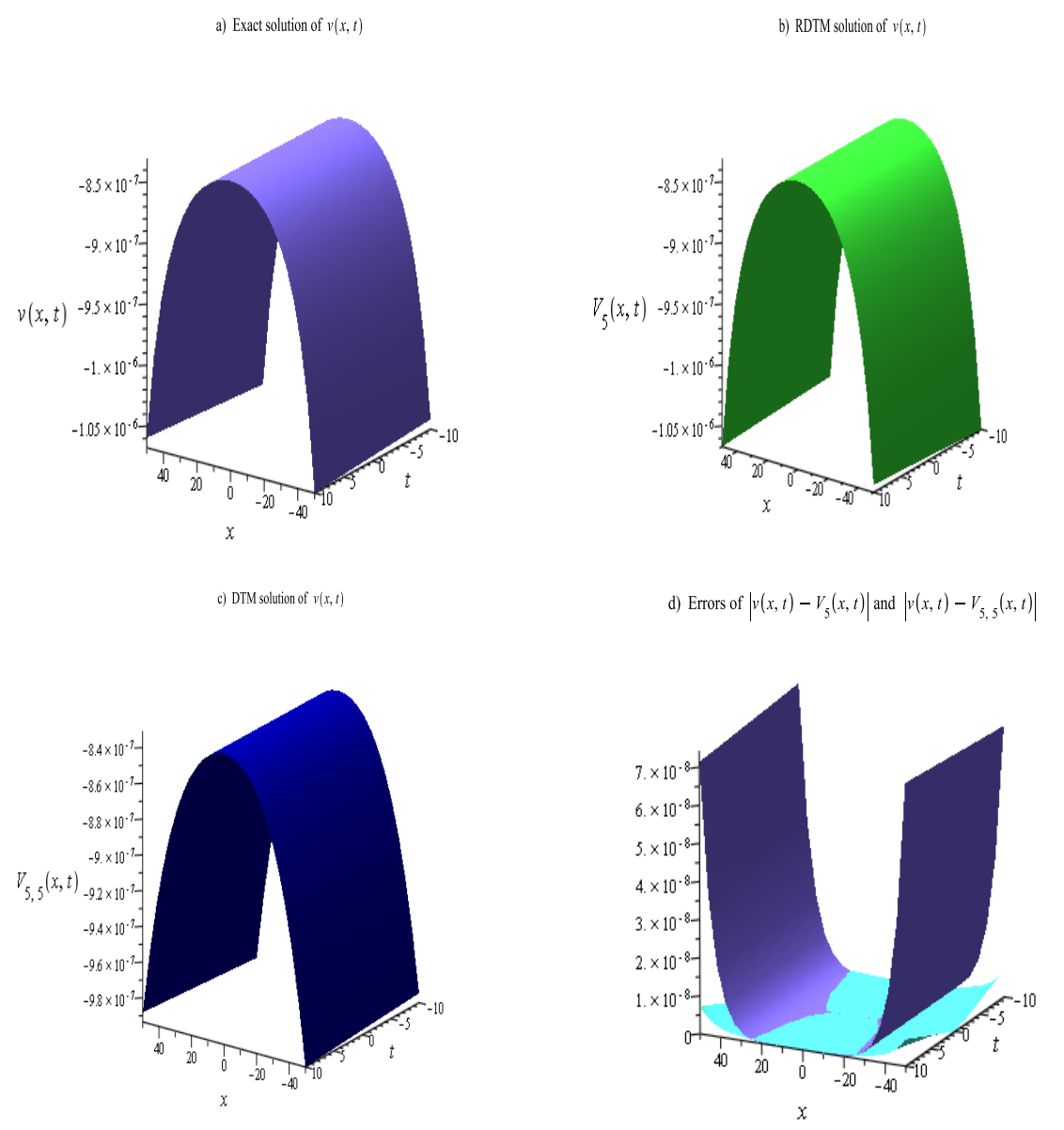

Fig. 4: Comparison between exact solution (3) and five steps RDTM, DTM solutions of $v(x, t)$ at $a_{0}=\frac{1}{2}, \alpha=\beta=0.02$. And comparison of errors d) MediumTurquoise-RDTM, SlateBlue-DTM.

\section{Conclusion}

In this paper, we consider the very famous physical problems coupled Ramani equation (1) to find two approximate traveling wave solutions by using DTM and RDTM. Moreover, we perfectly obtain approximate solutions of (1) compatible with exact solutions in [12]. In order to test efficiency, convergence and accuracy of DTM and RDTM, we perform the numerical values $a_{0}=1, \alpha=0.01, \beta=0.01$ and $a_{0}=\frac{3}{2}, \alpha=0.04, \beta=0.04$ and $a_{0}=\frac{3}{2}, \alpha=0.02$, $\beta=0.02$ in the seven step approximate solutions of eq. (1) which are shown in Table 3 to 8. Also, for $a_{0}=\frac{1}{2}, \alpha=0.03$, $\beta=0.03$ and $a_{0}=\frac{1}{2}, \alpha=0.02, \beta=0.02$, error rates for comparisons of exact [12] and seven step RDTM, DTM solutions are presented in Fig. (1) to (4). Furthermore, the CPU times of DTM and RDTM process are compared in Table 9. Results show that DTM and RDTM are efficient and powerful technique, but RDTM is more easier, fast and better than DTM. 
Table 7: Comparison of errors for seven step DTM and RDTM solutions with exact solution 2 at $a_{0}=\frac{3}{2}, \alpha=0.04$, $\beta=0.04$

\begin{tabular}{|c|c|c|c|c|}
\hline \multirow{2}{*}{$x$} & \multicolumn{2}{|c}{ Errors of RDTM } & \multicolumn{3}{c}{ Errors of DTM } \\
& $\left|u(x, t)-U_{7}(x)\right|$ & $\left|v(x, t)-V_{7}(x)\right|$ & $\left|u(x, t)-U_{7,7}(x, t)\right|$ & $\left|v(x, t)-V_{7,7}(x, t)\right|$ \\
\hline-50 & $1.03 \times 10^{-7}$ & $5.674312 \times 10^{-9}$ & 1041.757463 & $1.969313611 \times 10^{-4}$ \\
\hline-25 & $5.336 \times 10^{-6}$ & $2.2925246 \times 10^{-8}$ & 4.433682835 & $1.007732888 \times 10^{-6}$ \\
\hline-10 & $1.09 \times 10^{-5}$ & $1.9163592 \times 10^{-8}$ & 0.894570155 & $3.9147652 \times 10^{-8}$ \\
\hline 10 & $9.955 \times 10^{-6}$ & $1.7818539 \times 10^{-8}$ & 0.894281382 & $3.9334112 \times 10^{-8}$ \\
\hline 25 & $5.845 \times 10^{-6}$ & $2.365664 \times 10^{-8}$ & 4.503690346 & $7.2896423 \times 10^{-7}$ \\
\hline 50 & $3.31 \times 10^{-7}$ & $5.987977 \times 10^{-9}$ & 1047.990895 & $1.603518458 \times 10^{-4}$ \\
\hline
\end{tabular}

Table 8: Comparison of errors for seven step DTM and RDTM solutions with exact solution 3 at $a_{0}=\frac{3}{2}, \alpha=0.02$, $\beta=0.02$

\begin{tabular}{|c|c|c|c|c|}
\hline \multirow{2}{*}{$x$} & \multicolumn{2}{|c}{ Errors of RDTM } & \multicolumn{2}{c}{ Errors of DTM } \\
& $\left|u(x, t)-U_{7}(x)\right|$ & $\left|v(x, t)-V_{7}(x)\right|$ & $\left|u(x, t)-U_{7,7}(x, t)\right|$ & $\left|v(x, t)-V_{7,7}(x, t)\right|$ \\
\hline-50 & $1.6732 \times 10^{-5}$ & $1.3985587 \times 10^{-8}$ & 0.487909733 & $4.2968583 \times 10^{-8}$ \\
\hline-25 & $5.98 \times 10^{-7}$ & $1.35208 \times 10^{-9}$ & $4.6062085 \times 10^{-2}$ & $3.09447 \times 10^{-10}$ \\
\hline-10 & $1.33 \times 10^{-7}$ & $3.854221 \times 10^{-10}$ & $1.1065658 \times 10^{-2}$ & $3.51551 \times 10^{-11}$ \\
\hline 10 & $1.48 \times 10^{-7}$ & $3.721891 \times 10^{-10}$ & $1.1062022 \times 10^{-2}$ & $3.50481 \times 10^{-11}$ \\
\hline 25 & $6.2 \times 10^{-7}$ & $1.3290794 \times 10^{-9}$ & $4.6042264 \times 10^{-2}$ & $1.409608 \times 10^{-10}$ \\
\hline 50 & $1.4049 \times 10^{-5}$ & $1.4859454 \times 10^{-8}$ & 0.487320038 & $3.026153 \times 10^{-8}$ \\
\hline
\end{tabular}

Table 9: Comparison of CPU time for seven step DTM and RDTM solutions at $a_{0}=1, \alpha=0.03, \beta=0.03$

\begin{tabular}{|c|c|c|}
\hline Iteration numbers & CPU times for RDTM & CPU times for DTM \\
\hline 3 & 0.011 second & 16.297 second \\
\hline 5 & 0.015 second & 132.047 second \\
\hline 6 & 0.016 second & 307.406 second \\
\hline 7 & 0.026 second & 647.985 second \\
\hline 8 & 0.034 second & 1427.203 second \\
\hline
\end{tabular}

\section{Competing interests}

The authors declare that they have no competing interests.

\section{Authors' contributions}

All authors have contributed to all parts of the article. All authors read and approved the final manuscript.

\section{References}

[1] Ramani A., Inverse scattering, ordinary differential equations of Painleve-type and Hirotas bilinear formalism. In: Fourth International Conference Collective Phenomena, New York: Academy of Sciences (1981), p. 54. 
[2] Hirota R., Direct method of finding exact solutions of nonlinear evolution equations, In: Bullough R., Caudrey P., editors. Backlund transformations, Berlin, Springer (1980), p. 1157-75.

[3] Hu X. B., Wang D. L., Tam H. W., Lax pairs and backlund transformations for a coupled Ramani equation and its related system, Applied Mathematics Letters (2000), 13, 45-48.

[4] Zhao J. X., Tam H. W., Soliton solutions of a coupled Ramani equation, Appl Math Lett, 16 (2006), 307-313.

[5] Ablowitz M. J., Clarkson P. A., Solitons, nonlinear evolution equations and inverse scattering transform, Cambridge University press, 1990.

[6] Ito M., An extension of nonlinear evolution equations K-dV (mK-dV) type to higher order, J. Physc. Soc. Jpn. (1980), 49 (2), 771-778.

[7] Zhang H., New exact traveling wave solutions for some nonlinear evolution equations, Chaos Solitons and Fractals (2005), 26 (3), 921-5.

[8] Feng Z., Traveling solitary wave solutions to evolution equations with nonlinear terms any order, Chaos Solitons and Fractals (2003), 17 (5), 861-8.

[9] Malfliet W., Hereman W., The tanh method: I Exact solutions of nonlinear evolution and wave equations, Physica Sprica (1996), $54,569-75$.

[10] Li J., Existence of exact families of traveling wave solutions for the sixth-order Ramani equation and a coupled Ramani equation, Int. J. Bifurc. Chaos (2012), 22 (1), 125002.

[11] Nadjafikhah M., Shirvani-Sh V., Lie symmetries and conservation laws of the Hirota-Ramani equation, Commun. Nonlin. Sci. Numer. Simul. (2012), 17 (11), 4064-4073.

[12] Yusufoglu E., Bekir A., Exact solutions of coupled nonlinear evolution equations, Chaos Solitons and Fractals (2008), 37, 842-848.

[13] Wazwaz A. M., Triki H., Multiple soliton solutions for the sixth-order Ramani equation and a coupled Ramani equation, Applied Mathematics and Computation (2010), 216, 332-336.

[14] Wazwaz A. M., A coupled Ramani equation: multiple soliton solutions, J. Math. Chem. (2014), 52, 2133-2140.

[15] Jafarian A., Ghaderi P., Golmankaneh A. K., Baleanu D., Homotopy analysis method for solving coupled Ramani Equations, Rom. Journ. Phys. (2014), 59 (1-2), 26-35.

[16] Wazwaz A. M., Multiple soliton solutions for a new coupled Ramani equation, Physica Scripta (2011), 83, 015002.

[17] Zhou J. K., Differential Transformation and its Applications for Electrical Circuits. Huarjung University Press, Wuuhahn, China, (1986).

[18] Jang M. J., Chen C. L., Liu Y. C., On solving the initial value problem using the differential transformation method, Applied Mathematics and Computation (2000), 115, 145-160.

[19] Chen C. L., Ho S. H., Application of differential transformation to eigenvalue problems, Applied Mathematics and Computation (1996), 79, 173-188.

[20] Kurnaz A., Oturan G., Kiris M.E., N-Dimensional differential transformation method for solving PDEs, International Journal of Computer Mathematics (2005), 82(3), 369-380.

[21] Kurnaz A, Oturanc G., The differential transform approximation for the system of ordinary differential equations, Int J. Comput. Math. (2005), 82,709719.

[22] Keskin Y., Kurnaz A., Kiris M. E., Oturanc G., Approximate solutions of generalized pantograph equations by the differential transform method. International Journal of Nonlinear Sciences and Numerical Simulation (2011), 8(2), 159-164.

[23] Gubes M., Peker H. A., Oturanc G., Application of Differential transform method for El Nino Southern oscillation (ENSO) model with compared Adomian decomposition and variational iteration methods, J. Math. Comput. Sci. (2015), 15, 167-178.

[24] Abazari R., Borhanifar A., Numerical study of Burgers and coupled Burgers equations by differential transformation method, Comput Math Appl (2010), 59, 27112722.

[25] Srivastava V. K., Awasthi M. K., (1 + n)-Dimensional Burgers' equation and its analytical solution: A comparative study of HPM, ADM and DTM, Ain Shams Engineering Journal (2014), 5(2), 533-541.

[26] Abazari R., Abazari M., Numerical simulation of generalized Hirota-Satsuma coupled KdV equation by RDTM and comparison with DTM, Commun. Nonlin. Sci. Numer. Simul. (2012), 17, 619-629.

[27] Gubes M., Keskin Y., Oturanc G., Numerical solution of time-dependent Foam Drainage Equation (FDE), Computational Methods for Differential Equations (2015), 3(2), 111-122.

[28] Keskin Y., Oturanc G, Reduced Differential Transform Method for Partial Differential Equations, Int. J. Nonlinear Sci. and Num. Simulat. (2009), 10(6), 741-749.

[29] Keskin Y., PhD thesis, Selcuk University, 2010 (in Turkish). 
[30] Keskin Y., Oturanc G, Reduced Differential Transform Method for Solving Linear and Nonlinear Wave Equations, Iranian Journal of Science and Technology (2010), Trans A 34, A2.

[31] Keskin Y., Oturanc G., Reduced Differential Transform Method for fractional partial differential equations, Nonlinear Science Letters A (2010),1(2), 61-72.

[32] Rawashdeh M. S., An Efficient Approach for Time-Fractional Damped Burger and Time-Sharma-Tasso-Olver Equations Using the FRDTM, Appl. Math. Inf. Sci. (2015), 9(3), 1239-1246.

[33] Rawashdeh M. S., Obeidat N. A., On Finding Exact and Approximate Solutions to Some PDEs Using the Reduced Differential Transform Method, Appl. Math. Inf. Sci. (2014), 8(5), 2171-2176.

[34] Srivastava V. K., Awasthi M. K., Chaurasia R. K., Reduced differential transform method to solve two and three dimensional second order hyperbolic telegraph equations, Journal of King Saud University - Engineering Sciences (2016), in press.

[35] Srivastava V. K., Awasthi M. K., Chaurasia R. K. and Tamsir M., The Telegraph Equation and Its Solution by Reduced Differential Transform Method, Modelling and Simulation in Engineering (2013), Article ID 746351, 6 pages.

[36] Srivastava V. K., Mishra N., Kumar S., Singh B. K., Awasthi M. K., Reduced differential transform method for solving $(1+\mathrm{n})$ Dimensional Burgers' equation, Egyptian Journal of Basic and Applied Sciences (2014), 1(2), 115-119.

[37] Srivastava V. K., Awasthi M. K., Tamsir M., RDTM solution of Caputo time fractional-order hyperbolic telegraph equation, AIP Advances 3, 032142 (2013).

[38] Shukla H. S., Tamsir M., Srivastava V. K. and Kumar J., Approximate Analytical Solution of Time-fractional order CauchyReaction Diffusion equation, Computer Modeling in Engineering and Sciences (2014), 103(1), 1-17.

[39] Srivastava V. K., Awasthi M. K., Kumar S., Analytical approximations of two and three dimensional time-fractional telegraphic equation by reduced differential transform method, Egyptian Journal of Basic and Applied Sciences (2014), 1(1), 60-66. 